\title{
Ambiente, territorio y economía. Las actividades agroextractivas en el NEA
}

\author{
Prof. Aníbal Marcelo Mignone \\ Prof. Alejandra Helena Torre Geraldi \\ Centro de Geociencias Aplicadas- UNNE \\ Departamento de Geografía- UNNE \\ 11 de Octubre de 2012
}

\begin{abstract}
Presentado en:
Programa de Fortalecimiento Institucional de la Planificación Territorial Curso de Formación en Planificación y Ordenamiento del territorio para

Agentes Gubernamentales. Año 2012.

Módulo 2 - Problemas Territoriales

Actividades agro-extractivas, industria y organización del territorio
\end{abstract}


Esta presentación fue expuesta en el Programa de Fortalecimiento Institucional de la Planificación Territorial. Curso de Formación en Planificación y Ordenamiento del territorio para Agentes Gubernamentales. Año 2012. Módulo 2 - Problemas Territoriales: Actividades agro-extractivas, industria y organización del territorio. 11 de octubre de 2012.

\section{Objetivos del eje temático:}

- Introducir las tendencias actuales de la estructura productiva del país y la región.

- Analizar el manejo de los recursos naturales no renovables.

- Reflexionar sobre la incidencia de las políticas públicas en la problemática ambiental y socioeconómica de las economías regionales.

\section{Título:}

Ambiente, territorio y economía. Las actividades agroextractivas en el NEA

\section{Autores:}

Prof. Aníbal Marcelo Mignone, animarmig@hotmail.com

Prof. Alejandra Helena Torre Geraldi, torregeraldi@hotmail.com 


\section{Resumen}

Diversas prácticas, actores y procesos productivos están generando en la actualidad grandes transformaciones en las territorialidades del país. Asimismo, ciertas formas de producción y de manejo de los recursos naturales han instalado en la sociedad civil una renovada preocupación por la cuestión ambiental. Estas relaciones entre territorio, economía y ambiente adquieren cada vez más importancia en la planificación urbana y regional, especialmente en contextos de crecimiento macroeconómico y de fuerte dinamismo de las actividades productivas.

En lo que refiere al sector extractivo, el enfoque ortodoxo de la economía plantea la existencia de una suerte de "maldición" irreversible. Sin embargo, la experiencia indica que los impactos económicos y territoriales asociados a las actividades extractivas dependen de las políticas implementadas, en especial en cuanto a la modalidad de explotación y al destino de los ingresos derivados de la renta que generan. El desafío actual se plantea en torno a una política integral, articulada entre Nación y las provincias, que considere la importancia de los recursos como componente fundamentales en un proceso de desarrollo.

Algo similar ha ocurrido con la expansión de la "nueva agricultura", especialmente a partir de la expansión de la soja, cuyos mecanismos de producción/apropiación de renta y formas de manejo de los cultivos instalaron un fuerte debate sobre sus efectos económicos, socio-ambientales y territoriales, así como también respecto de las políticas que rigen en el sector. Por su parte, la industria ha sido uno de los sectores que más favorablemente reaccionó a los estímulos macroeconómicos tras la crisis de 2001/02 liderando una fase expansiva que permitió a varias ramas manufactureras recuperar el terreno perdido décadas atrás. Sin embargo, este cambio de tendencia parece no haber sido suficiente para modificar la matriz productiva heredada, equilibrar asimetrías de rentabilidad al interior del sector, ampliar y modernizar la capacidad instalada y superar algunas brechas territoriales. 


\section{Territorio - Ambiente - Economía}

- El hombre modifica el paisaje y con su trabajo va organizando el espacio que habita utilizando los recursos naturales para producir bienes de consumo, constituyendo así las bases de la economía.

- Mantobani (1991) considera que "los fenómenos ambientales resultan de la interacción entre los recursos naturales disponibles, la organización de la sociedad y la tecnología que se usa para su explotación", trae como consecuencia un cierto modelo de uso de los recursos naturales y un cierto modelo de uso y organización del territorio.

- En los diagnósticos económicos, la única alusión al territorio viene a ser aquella que sirve para nombrar el lugar en el que se desenvuelve la actividad económica. 


\section{Territorio - Ambiente - Economía}

Desde una perspectiva económica (Granda Caravajal, 2006), los entornos naturales tienen cuatro funciones:

- Proveen insumos al sistema productivo;

- Actúan como sumidero para los residuos resultantes de las actividades de producción y consumo;

- Constituyen el soporte de la vida (al facilitar fenómenos como la estabilidad ecosistémica y climática) y

- Suministran servicios de "amenidad", esto es, de esparcimiento y deleite.

La capacidad del ambiente natural para desempeñar estas funciones es finita. Tal finitud se relaciona con el nivel en que las actividades humanas lo afectan de modo irremediable (Granda Caravajal, 2006). 


\section{Visión de la economía del sistema económico}

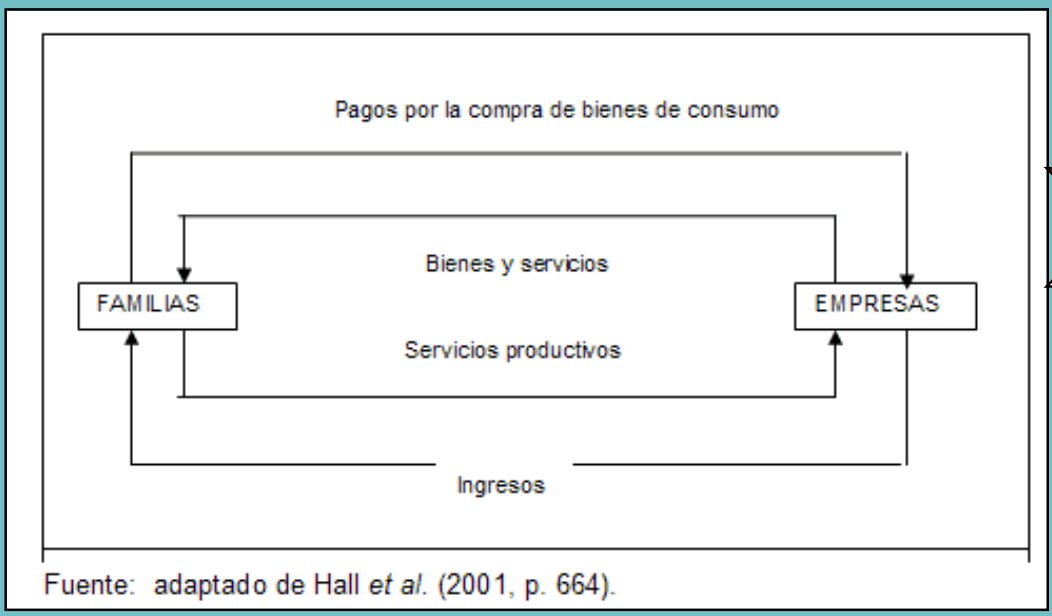

Sistema cerrado y aislado, en relación con el entorno físico y social

Sistema equilibrado: el valor que se liquida mediante el consumo

\section{Visión biofísica del sistema económico}

Sistema abierto: intercambiando
energía y materiales con su entorno
Sistema desequilibrado: sujetos a la
flecha unidireccional del tiempo, -ley
de la entropía-, y cerrando los ciclos y
transformando los residuos en nuevas
reservas orgánicas

Fuente: Extraído de Granda Caravajal (2006) y de Delgado Cabeza (2006)

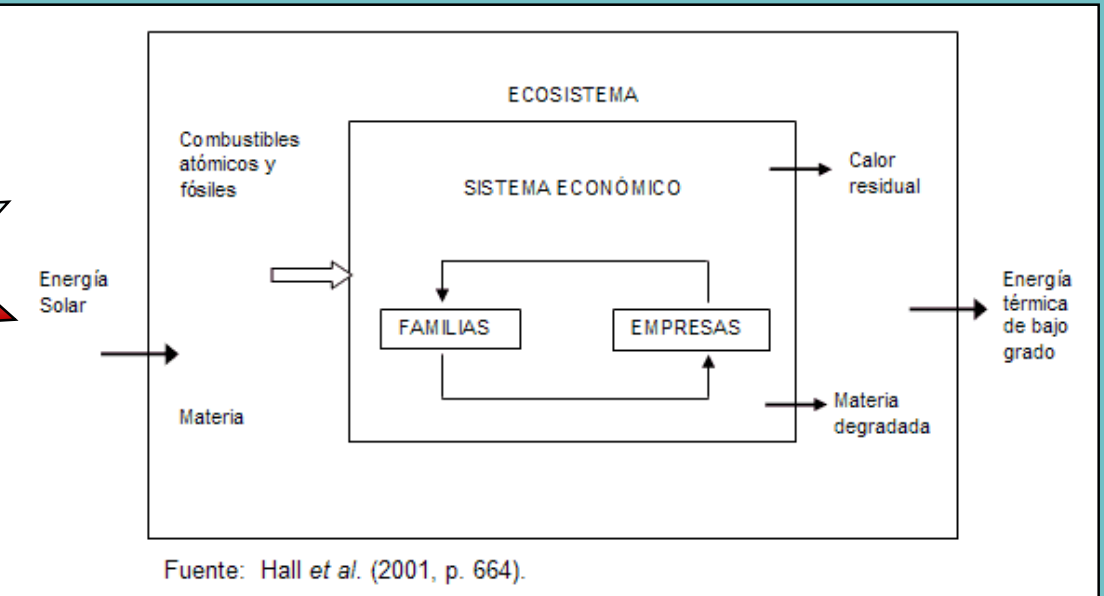

Publicado en formato digital: Prof. Aníbal Marcelo Mignone y Prof. Alejandra Helena Torre Geraldi. AMBIENTE, TERRITORIO Y ECONOMÍA. LAS ACTIVIDADES AGROEXTRACTIVAS EN EL NEA. Revista Geográfica Digital. IGUNNE. Facultad de Humanidades. UNNE. Año 13. № 26. Julio - Diciembre 2016. Resistencia, Chaco. En: http://hum.unne.edu.ar/revistas/geoweb/default.htm 


\section{Territorio - Ambiente - Economía}

- Siendo el territorio un componente fundamental de todo proceso económico, su gestión se maneja y se justifica desde una economía cuyas reglas de funcionamiento van en dirección contraria a las que reclama el mantenimiento del entorno físico.

- Según las actividades económicas a que se dediquen los territorios, el papel que jueguen dentro del sistema, y las relaciones que se establezcan entre ellos, va a existir un deterioro que adquiere distintos grados y diferentes implicaciones.

- El progreso ha conducido a una crisis ecológica, a desequilibrios interterritoriales extremos en todas las escalas.

- Se han rebasado límites éticos en aras del progreso y del desarrollo económico, la destrucción de recursos naturales, empleo de tecnologías dañinas para la sociedad.

- La planificación territorial y la ordenación del territorio son el marco idóneo para la incorporación del paradigma ambiental en los programas de desarrollo territorial (Cuesta Aguilar, 2006). 
Friedman y Weaber (1979) consideran que "el progreso económico de un territorio solo es posible cuando las empresas y los demás actores del territorio interactúan entre sí, se organizan y realizan sus inversiones dirigidas a desarrollar la economía y la sociedad local", atendiendo también, a cuestiones ambientales. 


\section{LAS ACTIVIDADES AGROPECUARIAS DEL NEA}

- En el Oriente de la región, en el ámbito húmedo de Misiones y el noreste de Corrientes: yerba mate, te y tung.
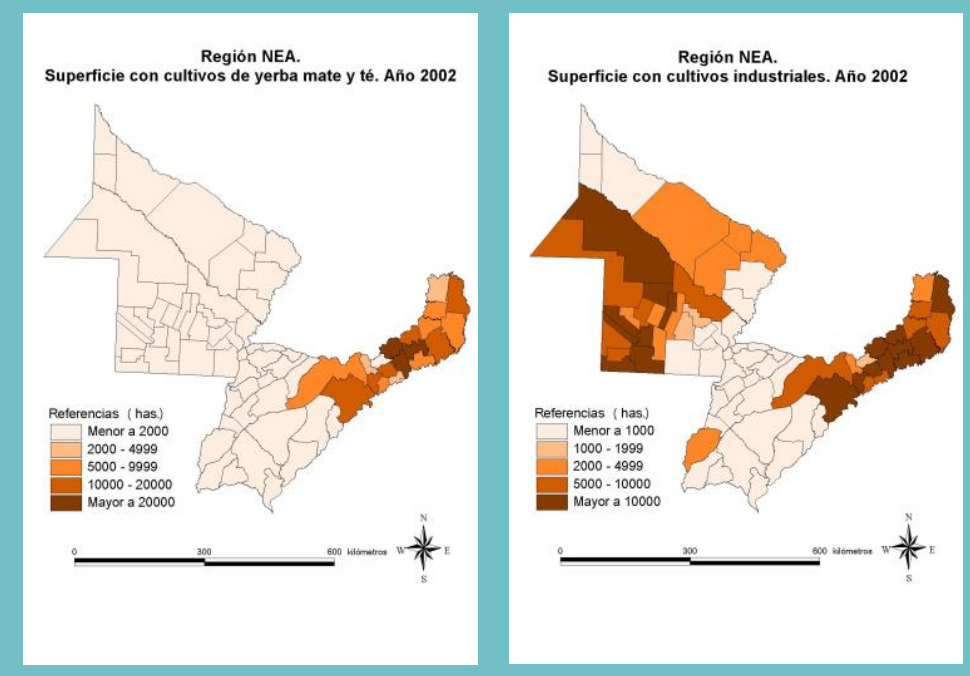

- Planicie centro chaqueña (centro y oeste de Chaco y Formosa) los cultivos anuales de secano: algodón, trigo, maíz, girasol.
En el área intermedia Occidente de Corrientes y en el sector húmedo oriental de Chaco y Formosa: tenemos núcleos de estancias para la cría de vacunos en grandes extensiones, alternando con policultivos a lo largo del eje Paraná- Paraguay, citrus y tabaco en el occidente correntino y arroz en el sur-sureste de Corrientes.
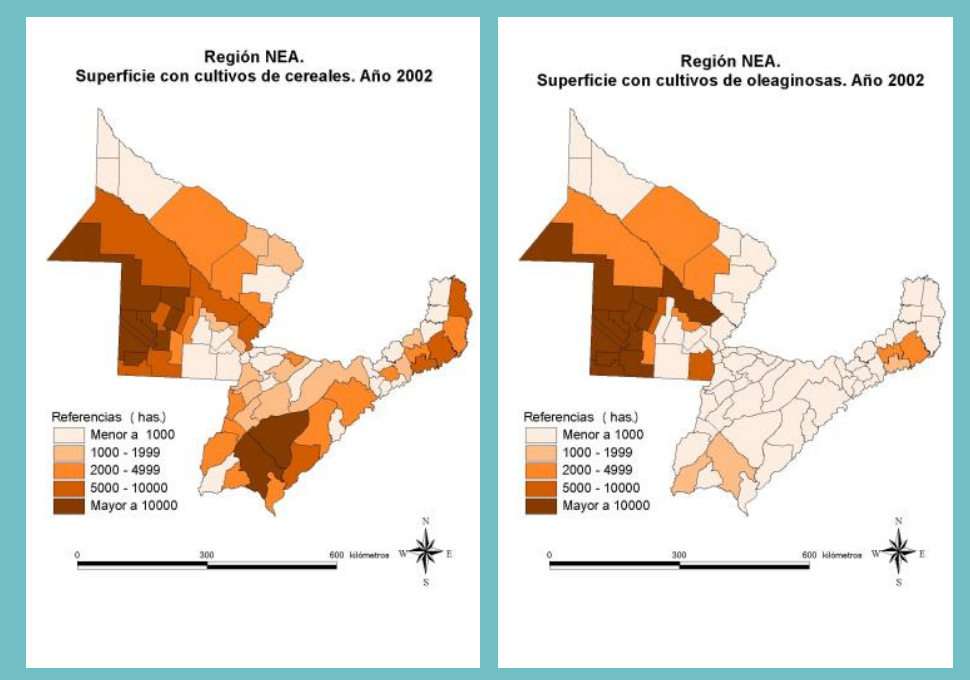

Fuente: Elaboración propia según INDEC. Censo Nacional Agropecuario 2002 


\section{Características de las actividades agropecuarias del NEA}

El sector agrario regional alberga dos modalidades diferentes de producción:

- Plantaciones perennes en Corrientes y Misiones.

- Cultivos anuales en Chaco y Formosa.

- Ganadería extensiva adaptada a las exigencias del ganado subtropical.

- Localización en un clima de transición la priva de beneficios de la especialización productiva -agropecuaria del área situada al sur de la región (Bruniard, 1987). 


\section{Características de las actividades agropecuarias del NEA}

- Marcada bipolaridad de la estructura agraria regional: predominio de grandes fundos y de minifundios (Valenzuela, 2006).

- Debilidad del mercado interno regional y falta de complementariedad (Schweitzer, 2004).

$r$

- Las provincias del Nea conforman los "enclaves de drenaje" (Ferraro, 1973) por:

- Depender de las demandas alóctonas o movido por energías extrarregionales.

- No existe una aceptable integración agroindustrial.

- El destino de la producción es el mercado nacional (Bruniard, 1987). 


\section{La Pampeanización del NEA a partir de la década de 1960}

\section{Caracteres}

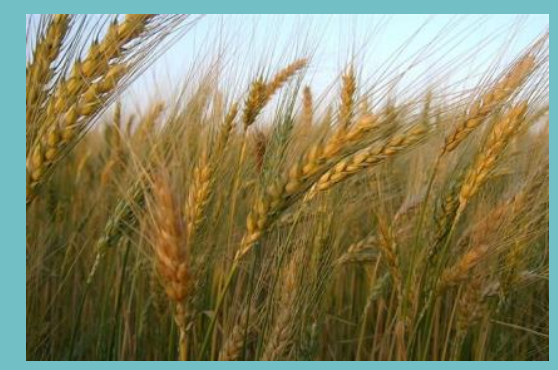

Reemplazo de cultivos industriales por actividades agrarias propias de la región pampeana (cultivos alternativos: trigo, soja, girasol, sorgo, maíz y actividad pecuaria)

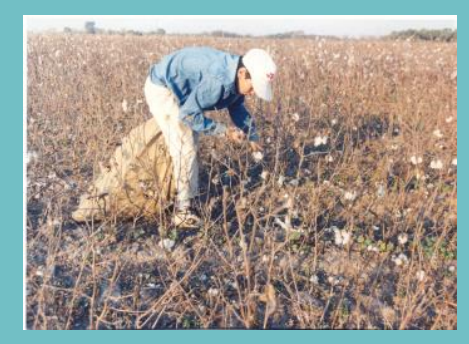

\section{Expansión sobre territorios tradicionales}

\section{Efectos}

\section{Mano de obra. Migración rururbana Deterioro ambiental Disminución del valor agregado de la producción}




\section{CAMBIOS AGROPRODUCTIVOS EN LA REGIÓN NEA A PARTIR DE LA DÉCADA DE 1990}




\section{La Globalización \\ (a partir de 1990)}

\section{Desregulación de las actividades agropecuarias}

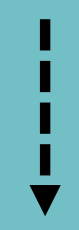

\section{Supresión de organismos reguladores}

Precarización del mercado laboral

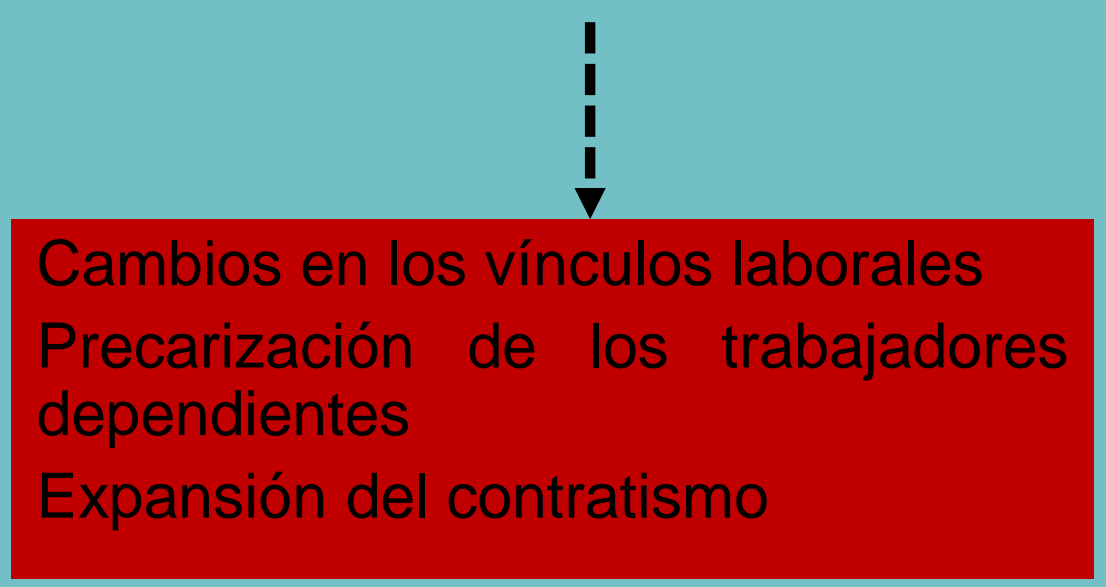

\section{Se consolida el modelo "extractivo" en torno a las oleaginosas}




\section{EXPANSIÓN DE LA FRONTERA AGROPECUARIA EN EL NEA}

Fundamentada en:

- Proceso de "pampeanización" debido al incremento de la superficie cultivada de soja.

- Incremento de la mecanización.

- Introducción de mejoras genéticas.

- (OGM) y uso de fertilizantes y biocidas.

- Aumento en el tamaño de las explotaciones (mayor superficie de unidad productiva por concentración).

- Alta inversión de capital extrarregional. 


\section{EXPANSIÓN DE LA FRONTERA AGROPECUARIA EN EL NEA}

- Se conforma un territorio estructurado sobre la base de grandes explotaciones cuyo actor principal es la empresa agropecuaria (Bolsi et. al., 2009).

- Los empresarios pueden adquirir grandes extensiones de tierra e incorporar los paquetes tecnológicos.

- Las grande empresas incorporan parcialmente elementos de la agricultura conservacionista y un escaso desarrollo de la agricultura sustentable (Morello y Pengue, 2007).

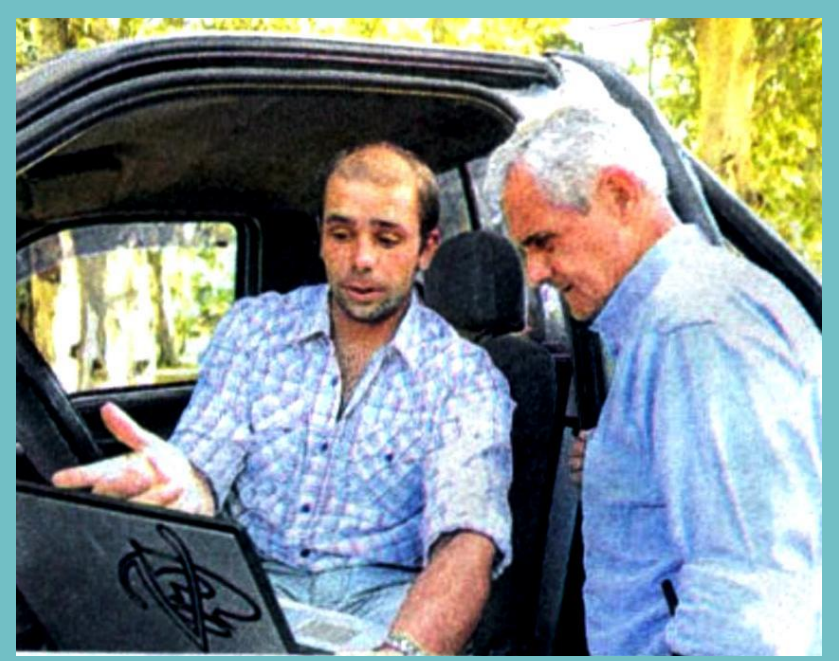

Fuente: Rural Revista.11 de febrero de 2012-:35 


\section{EXPANSIÓN DE LA FRONTERA AGROPECUARIA EN EL NEA}

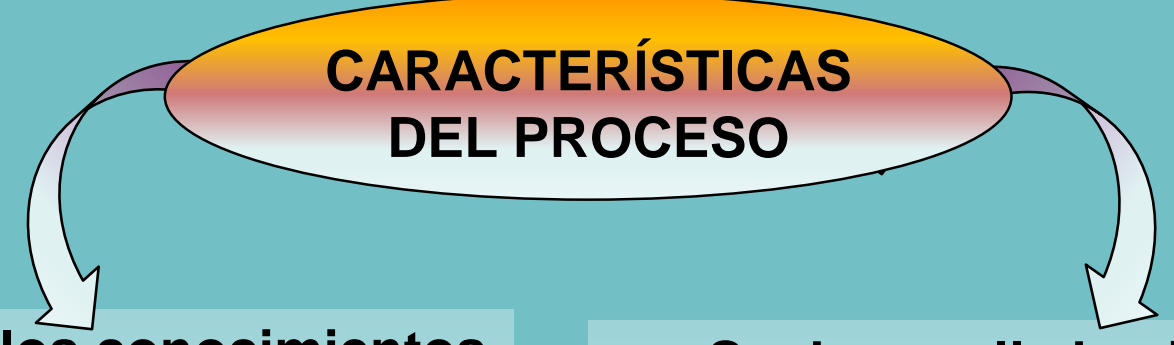

- Poco respeto por los conocimientos ecológicos de las etnias locales.

- Madariaga (2000) destaca la pobreza de resultados en materia de transformaciones y avances estructurales significativos de las áreas de expansión.

- Incremento de los niveles de desocupación en áreas rurales (agrarias), por reemplazo de mano de obra por maquinarias.
- Se desarrolla hacia los sectores marginales del sudoeste y oeste donde existe:

*Balance hídrico negativo. *Baja materia orgánica del suelo

*Suelos salino-alcalinos o de clase III, IV o V

- Fue factible por factores climáticos: períodos húmedos o de excesos hídricos durante la década de 1990. 


\section{EL CLIMA Y SU INFLUENCIA EN LAS ACTIVIDADES AGROPECUARIAS}

- A partir de la década de 1960 se observa un incremento en el monto de las precipitaciones en el NEA.

- Se produce un desplazamiento de las isohietas hacia el oeste, especialmente la de $800 \mathrm{~mm}$.

- No hay una tendencia definida y continua, ascendente 0 descendente de los montos pluviométricos, por lo tanto no se trata de tendencias permanentes sino de ciclos naturales con fases temporalmente positivas o negativas cuyo origen parece estar asociado a factores dinámicos ligados con la circulación general de la atmósfera (Bruniard, 1990; Pérez, 2009). 


\section{Algunas consecuencias de la expansión de la frontera agropecuaria}

\section{En lo ambiental}

- Daños al medio ambiente: contaminación de agua, suelo y aire.

- Afecta la biodiversidad (disminución o desaparición de especies endémicas).

- Reducción de superficie de los bosques nativos.

- Quema de la biomasa.

- Erosión y voladuras de suelos.

- Los costos (externalidades) no se incluyen en las cuentas de ganancias 0 pérdidas.

\section{En la población}

- Desplazamiento de población de áreas rurales a espacios urbanos.

- Impacto en la sostenibilidad del sector rural.

- Afecta a las ciudades en cuanto al tipo de función que cumplen.

- Afecta los cinturones periurbanos (influencia de las áreas fumigadas). 


\section{EL USO DE LA TECNOLOGÍA. APLICACIÓN EN LOS PRODUCTORES ALGODONEROS CHAQUEÑOS \\ (Valenzuela y Scavo, 2007)}

Los pequeños productores:

- No aplican tecnología de avanzada; en casos excepcionales, utilizan plaguicidas.

- Incorporan las prácticas culturales si los extensionistas pertenecientes a organismos oficiales brindan apoyo directo.

- La cosecha se efectúa manualmente y las labores de suelo se realizan con tracción a sangre.

- Poseen escasa maquinarias y de baja potencia.
Los medianos y grandes productores:

- Aplican nuevas tecnologías de avanzada, laboreos técnicos y mecánicos y realizan control de plagas con fitoterápicos.

- La cosecha se ejecuta mecánicamente. Cuentan con maquinarias renovadas $y / 0$ de gran potencia.

- Realizan cursos de capacitación y perfeccionamiento. 


\section{Tecnología y problemas ambientales}

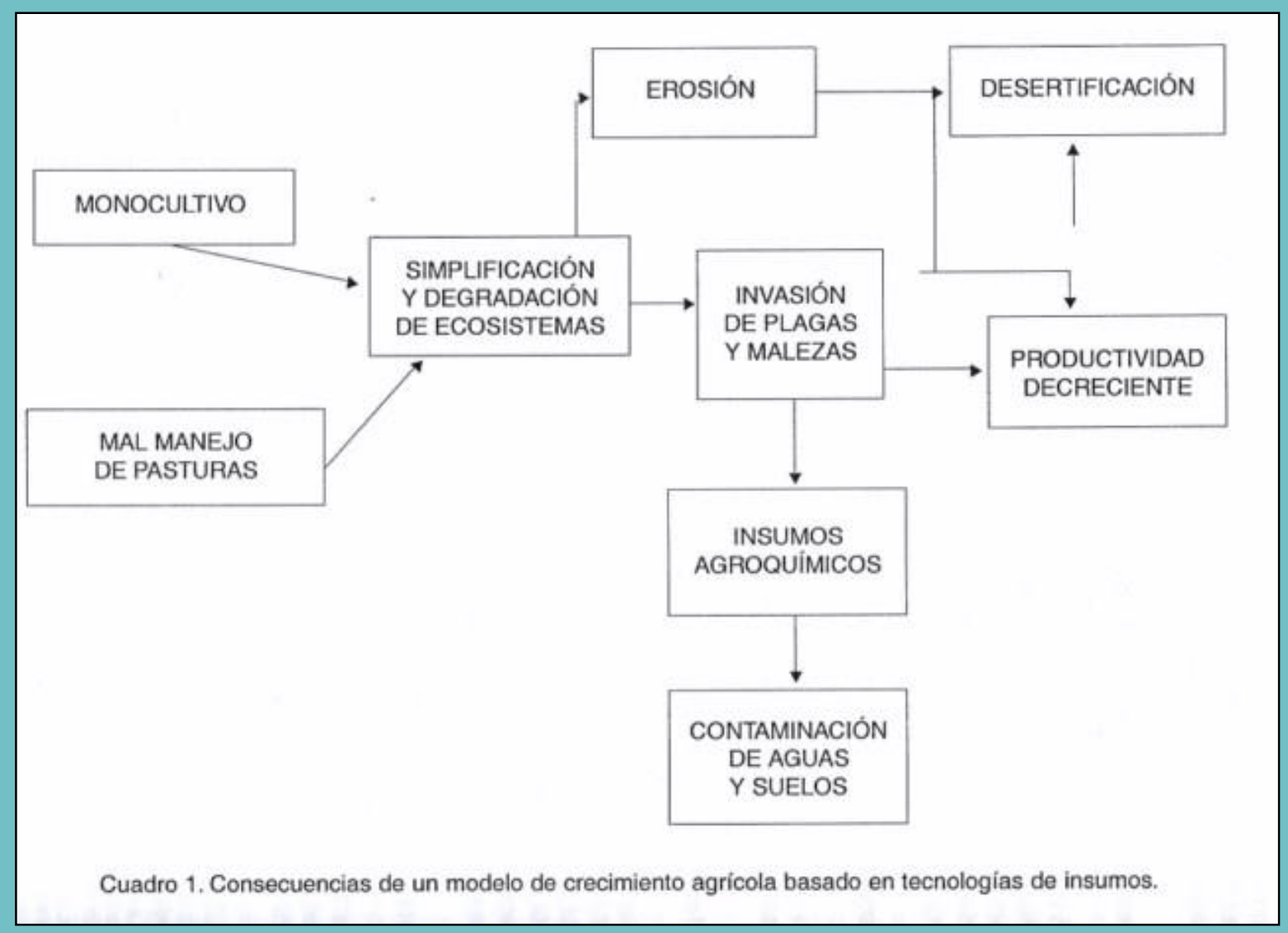

Fuente: Cervio, 2008:128

Publicado en formato digital: Prof. Aníbal Marcelo Mignone y Prof. Alejandra Helena Torre Geraldi. AMBIENTE, TERRITORIO Y ECONOMÍA. LAS ACTIVIDADES AGROEXTRACTIVAS EN EL NEA. Revista Geográfica Digital. IGUNNE. Facultad de Humanidades. UNNE. Año 13. № 26. Julio - Diciembre 2016. Resistencia, Chaco. En: http://hum.unne.edu.ar/revistas/geoweb/default.htm 


\section{Tecnología y problemas ambientales}

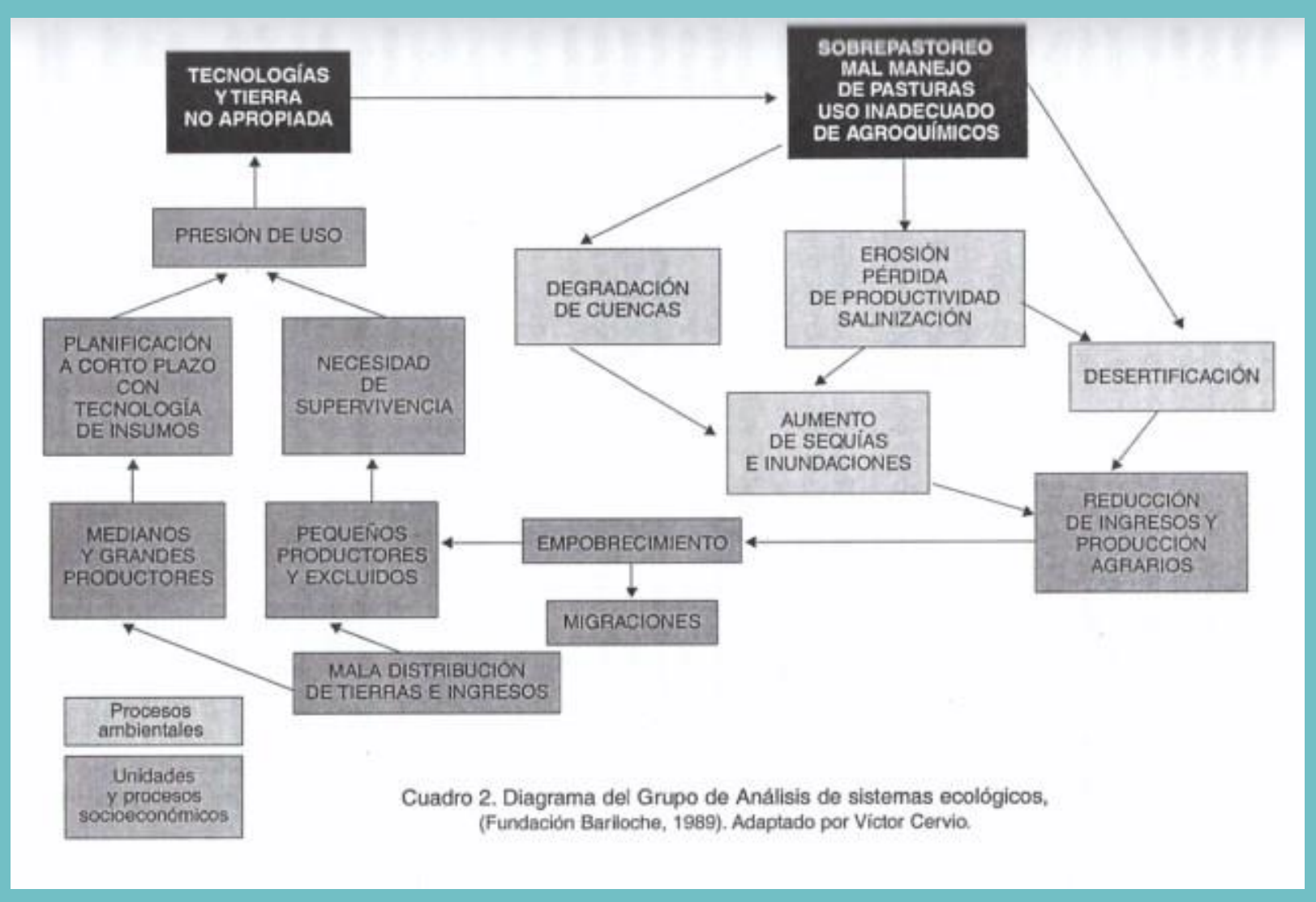

Fuente: Cervio, 2008:131

Publicado en formato digital: Prof. Aníbal Marcelo Mignone y Prof. Alejandra Helena Torre Geraldi. AMBIENTE, TERRITORIO Y ECONOMÍA. LAS ACTIVIDADES AGROEXTRACTIVAS EN EL NEA. Revista Geográfica Digital. IGUNNE. Facultad de Humanidades. UNNE. Año 13. № 26. Julio - Diciembre 2016. Resistencia, Chaco. En: http://hum.unne.edu.ar/revistas/geoweb/default.htm 


\section{Características de los factores que influyen en la sustentabilidad ambiental según el tipo de productores}

\begin{tabular}{|l|c|c|}
\hline \multicolumn{1}{|c|}{ Variables } & Capitalistas & Campesinos \\
\hline Coherencia ecológica & Mediana & Muy alta \\
\hline Estabilidad socioestructural & Mediana & Mediana \\
\hline Complejidad infraestructural & Alta & Baja \\
\hline Estabilidad económico financiera & Relativamente alta & Muy baja \\
\hline Incertidumbre y riesgo & Muy altos & Muy bajos \\
\hline
\end{tabular}

Fuente: Gligo, 1998 


\section{Dificultades de los pequeños productores}

- Tenencia precaria de la tierra.

- Endeudamiento crónico.

- Imposibilidad de acceso al crédito o de incorporar tecnologías de altos insumos o tecnologías híbridas.

- Presionados por grupos económicos que quieren acceder a los territorios para la producción agropecuaria intensiva.

- Desde mediados de los '90 tenemos cambios en los vínculos laborales por precarización creciente de los trabajadores del agro o expansión de los contratos temporales.

- Ausencia sistemática del estado (Rofman, 1999).

- Desplazamiento hacia áreas urbanas. 


\section{LA EXPANSIÓN DE LAS OLEAGINOSAS}

Se consolida el modelo extractivo de explotación de las oleaginosas desde mediados de la década de 1990.

Según Valenzuela (2006) se incrementa la superficie sojera, de acuerdo con diferentes estrategias:

- Sembradoras de siembra directa o la organización de los pools de siembra.

-Interesa el control de la tierra más que la propiedad.

- La estrategia productiva ahorra mano de obra. 


\section{LA EXPANSIÓN DE LAS OLEAGINOSAS}

- Desplaza a los pequeños productores.

- Tiende a eliminar al agricultor como sujeto social.

- Desplaza buena parte de las estructuras campesinas asociadas con la explotación tradicional. 
Revista Geográfica Digital. IGUNNE. Facultad de Humanidades. UNNE. Año 13. № 26. Julio -Diciembre 2016. ISSN 1668-5180. Resistencia, Chaco

\section{BIBLIOGRAFÍA}

- Arrillaga, Hugo; Lucila Grand; Valentina Locher y Gabriela Busso. Cambios en la matriz productiva del sector agropecuario y dinámica poblacional en la región pampeana. En Internet: http://www.econ.uba.ar/planfenix/novedades/Area\%20IV/Cambios\%20en\%20la\%20matriz\%20-\%20Arrillada-Grand-Locher-Busso.pdf. Visitado el 15 de septiembre de 2012.

Bolsi Alfredo, Fernando Longhi, Norma Meichtry y Pablo Paolasso. 2009. "El ámbito regional. Interrogantes y conjeturas. El territorio del Norte Grande Argentino como contexto de la pobreza. En Geografía de la Pobreza en el Norte Grande Argentino. Alfredo Bolsi y Pablo Paolasso (comp.). 1ํㅡㄹición, Tucumán: el autor. PP 123-171. Bruniard, Enrique. 1987. Geografía del Nordeste Argentino. Buenos Aires, SENOC-OIKOS.

Bruniard, Enrique. 1987. Geografía del Nordeste Argentino. SENOC, Bueno Aires.

Cervio, Víctor. 2008. Los recursos no son tan naturales. En: Agrosistemas: impacto ambiental y sostenibilidad. Giuffre, Lidia (coord.) Universidad de Buenos Aires, $1^{\circ}$ Edición. Pp.123-138.

Clarín. Rural Revista. Edición № 28. Junio-Julio 2012. P27

Cuesta Aguilar, María José. 2006. Ordenación del territorio, medio ambiente y globalización: reflexiones desde la Geografía regional al nuevo contexto socio-económico. Boletín de la A.G.E. № 42. Pp 255- 269. En Internet: dialnet.uniroja.es/descarga/articulo/2219479.pdf

Gligo, Nicolo. 1998/99. Desarrollo económico recursos naturales y productivos y la sustentabilidad regional. En "Seminario sobre desarrollo sustentable, políticas y acciones. Maestría en Gestión Ambiental". Facultad de Arquitectura y Urbanismo, UNNE.

Granda Carvajal, C. 2006. "Relaciones Medio Ambiente-Economía. La necesidad de una nueva mirada". En: Contribuciones a la Economía, agosto 2006. En Internet: http://www. eumed. net/ce. Visitado 12 de septiembre de 2012.

Hoffmann, J. A. 1988. "Las Variaciones Climáticas Ocurridas en la Argentina desde Fines del Siglo Pasado hasta el Presente". En: El Deterioro del Ambiente en la Argentina. Buenos Aires, FECIC

INDEC. S/f. Censo Nacional Agropecuario 2002. En Internet: www.indec.gov.ar. Visitado el 10 de septiembre de 2012.

Lenardón, A, Maitre M,I., Lorenzatti E. 2000. Plaguicidas organoclorados en leche materna en Santa Fe. Acta Toxicologica Argentina, 2000-8(1), 2-4.

Maggi, J. 2006). Cáncer y malformaciones por la contaminacion en Santa Fe. En Internet: http://argentina.indy media.org/news/2006

Minetti, J. y Vargas, W. 1997. "Trends and Jumps in the annual precipitation in South America, south of the $15^{\circ}$ Sur". En: Atmósfera. México, Vol. 11.

Morello, Jorge y Walter Pengue. 2007. "Procesos de transformación en las áreas de borde agropecuario: ¿una agricultura sostenible?”. En: Revista Encrucijadas, № 41, UBA, Buenos Aires.

Pérez, María Emilia. 2009. "El comportamiento temporal y espacial de los montos estacionales de precipitación en el Nordeste Argentino - 1931/2005". En Revista Geográfica Digital. IGUNNE. Fac. de Humanidades. Año 6, № 11. Enero- junio de 2009. En Internet:

http://hum.unne.edu.ar/revistas/geoweb/Geo11/archivos/meperez09. pdf. Visitado el 14 de septiembre de 2012.

Preumayr, Fernando. "Nueva realidad y nuevos desafíos". En: Rural revista. Clarín. 11 de febrero de 2012:: $34-35$.

Rofman, Alejandro y otros. 2008. "Subordinación productiva en las economías regionales de la posconvertibilidad", en: Realidad Económica, № 240.

Rofman, Alejandro. 1999. "Modernización productiva y exclusión social en las economías regionales". En: Realidad Económica № 162. IADE, Buenos Aires.

Schweitzer, Alejandro. 2004. ¿Uno o varios NEA? Regiones y territorios en el espacio del Nordeste Argentino. En: Crisis fiscal, mercado de trabajo y nuevas territorialidades en el nordeste argentino. Panaia, Marta (coord.) La Colmena, Buenos Aires. Pp 41-62.

Torre Geraldi, Alejandra. 2005. "La expansión de la soja en la provincia del Chaco y su inserción en el cluster oleaginoso argentino a fines del siglo XX". En Actas de XXV Encuentro de Geohistoria Regional. Organizado por el Gobierno de la Provincia de Corrientes. 25 a 27 de agosto de 2005. Versión CD Rom.

Torre Geraldi, Alejandra. 2008. "Cambios productivos en sector agrícola de la Provincia del Chaco: expansión de la soja y su impacto socioeconómico a fines del siglo XX". En II Jornadas Nacionales de Investigadores de las Economías Regionales y IX Encuentro Nacional de la Red de Economías Regionales en el Marco del Plan Fénix. UNCPBA; Proyecto Estratégico de la Universidad de Buenos Aires PLAN FENIX; Centro de Estudios Urbanos y Regionales- CONICET. 18 y 19 de septiembre de 2008. Tandil, Buenos Aires.

Torre Geraldi, Alejandra. 2008. "Impacto socioambiental de la expansión de soja en el Chaco, 1998-2008". En: VI Jornadas de Investigación y Debate "Territorio, Poder e Identidad en el Agro Argentino". Universidad Nacional de Quilmes. Versión en CD-Rom.

Torre Geraldi, Alejandra. 2010. Conflictos socioambientales en la provincia del Chaco: el caso de los cultivos de soja y arroz en el primer decenio del siglo XXI". En: VII Jornadas en Investigación y Debate. "Conflictos rurales en la Argentina del Bicentenario. Significado, alcances y proyecciones. Universidad Nacional de Quilmes. 19 al 21 de mayo de 2010. Bernal, Buenos Aires.

Valenzuela, Cristina y Ángel Scavo. 2009. La trama territorial del algodón en el Chaco. Una visión multiescalar. Editorial La Colmena.

Valenzuela, Cristina. 2006. Transformaciones agrarias y desarrollo regional en el Nordeste Argentino. Una visión geográfica del siglo XX. Editorial La Colmena.

Publicado en formato digital: Prof. Aníbal Marcelo Mignone y Prof. Alejandra Helena Torre Geraldi. AMBIENTE, TERRITORIO Y ECONOMÍA. LAS ACTIVIDADES AGROEXTRACTIVAS EN EL NEA. Revista Geográfica Digital. IGUNNE. Facultad de Humanidades. UNNE. Año 13. № 26. Julio - Diciembre 2016. Resistencia, Chaco. En: http://hum.unne.edu.ar/revistas/geoweb/default.htm 